\title{
Dual Monitoring Tracking System for the Protection of Children
}

\author{
Jong Chan Kim*, Oh Hoon Cho* and Eung Kon Kim* \\ *Department of Computer Science, Sunchon National University, \\ 315 Maegok Suncheon, Jeonnam, Korea \\ seaghost@sunchon.ac.kr
}

\begin{abstract}
According to the statistics of the national police agency, children are accounted for the high percentage of the reports about damage from the crime of kidnapping. Child protection means all types of services and programs that society or adults can do for children in order to promote their healthy growth and development process. The balance and harmony in surveillance and privacy protection is one of today's sensitive issues. Current CCTV is permitted on the premise of the clear notification, when used in the legal purposes of policing, security, transportation, and etc. In this paper, for the prevention of child-related crime, wide-area surveillance cameras and PTZ cameras with high magnification were used in schools, apartments, and playgrounds. The system tracking and monitoring abnormal face using the AdaBoost algorithm was proposed. In the method for detecting abnormal face by the use of methods to move the center of the whole image, 95.5\% of the abnormal facial recognition rate and the average speed of 17 frames of abnormal facial recognition were obtained. It is considered that this system will help prevent crime through the detection and tracking the faces of intelligent offenders who are emerging as a serious social problem.
\end{abstract}

Keywords: Child Protection, Dual Monitoring, Tracing System, AdaBoost Algorithm

\section{Introduction}

In order to prevent child abduction and relatedcrimes, it is requested to install widearea surveillance cameras and cameras with high magnification around schools, apartments, and playgrounds. Monitoring is the interaction of high-pressure atmosphere that require morality of the monitored mainly in public places or residential areas or shops.

But the current CCTV (Closed-Circuit Television) is permitted on the premise of the clear notification, when used in the legal purposes of policing, security, transportation, and etc. The case of monitoring children is aimed to complement the child's incompleteness rather than the concept of CCTV for the purposes of security, so the new approach is needed. The types of criminal offenders consist of first-time offenders and at $36.8 \%$ and $63.2 \%$ recidivist; two recidivism rates appeared to be about two times higher. Among these means to prevent crimes against children and women, the most rapidly developing one is the image analysis system with CCTV[1][2]. In 1997, U.S. Department of Defense Advanced Research Projects Agency (DARPA) information systems developed the technology of office video surveillance cameras and monitoring (VSAM) for the development of automated image understanding technology with the purpose of its use in the future urban and battlefield surveillance applications, and monitored a wide range of areas.

The existing security system, built on saving the image, has its advantages in terms of easy use and low cost maintenance, but there are the defects that the video cameras should be installed in the each main point, and the images should be recorded on the VCR (Video 
Cassette Recorder) or DVR (Digital Video Recorder), and then reviewed after the event has occurred. And to help prevent crime, managers should directly the watch the monitor for 24 hours a day. Because of this inconvenience, it cannot provide an effective response to criminal cases after school or on weekends or holidays, and culprits abuse this time range. As a result, it was difficult to provide a preliminary prevention of crime. In recent years, automatic security surveillance became possible to some extent by combining motion detection (motion detection) sensor with DVR and IP (Internet Protocol) cameras, but even in the case of physical sensor detection, its performance is not enough to meet expectations for building the complete automation system. Accordingly, it has increased the necessity of the integrating the development of intelligent video surveillance systems and software implementing the intelligent scene analysis technologies, which is one of the alternatives that can overcome the limitations of the existing video security systems and motion-sensing technology.

In this paper, to prevent growing crime againnst children, we proposed a dual monitoring tracking system which tracks the abnormal person using wide-area surveillance cameras and PTZ cameras with the high magnification. As the test shows 95.5\% recognition rate of abnormal face and 17 frames of the average track speed were achieved. Using this system, abnormal situations can be tracked and monitored through notification services and smart phones in the time of their occurrence. It would also be more helpful in child protection and crime prevention against intelligent criminals.

\section{Related Works}

\subsection{Trends in Child Monitoring System}

Robot Laboratory at Carnegie Mellon University and the configuration team of the Sarnoff Corporation developed the End to End Test System for the detection and tracking in real time from still to moving objects. The study of the technology sector, including object tracking, human gait analysis, simple multi-agent activities, and real-time data transfer, data logging and visualization recognition of dynamic scenes and the geometric method to recognize human activity, was performed[3].

Child monitoring product groups are classified as Winbel baby, Phillips baby, and Teddy cam. The first is the Winbel baby monitoring products group in which the camera feature hsa added sound interaction. The second is the Philips group, like baby monitors released by Philips, in which the sound interactions are possible. Temperatures or the situation around can be fed back via an icon and text, but it also has drawbacks through which the relief situation cannot be seen. The third type has a child-friendly teddy bear appearance, which makes it possible to perform monitoring of active play type. These groups set the details of functions, depending on the features of the specific target age group, but commonly it is difficult for them to monitor-blind spots, and the communication between the monitor and the monitored is also vulnerable. Figure 1 shows the commercially available child monitoring products. 
A

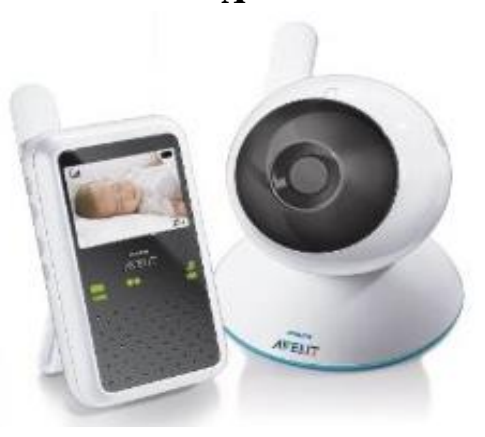

B

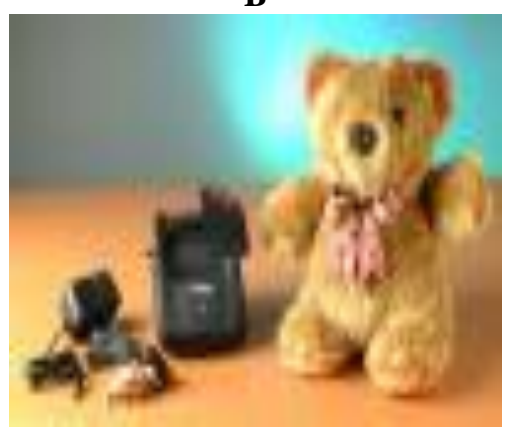

\section{Figure 1. Child Monitoring Product Groups. A: Phillips Baby Monitor, B: Teddy Cam}

\subsection{Haar-like Feature}

The face image input from the camera is much affected by the various external environmental factors. Environmental factors affect detection methods in which robust and fast computation is required. As the features for detection, Haar-like feature was used as the set of characteristics for the object detection. Haar-like feature can be computed quickly using the integral image. Using this Haar-like feature and the integral image, they can be used effectively in real-time object detection where the reduction of complex operations, and a high detection performance is required.

Figure 2 shows the representative Haar-like features, which are classified as edge features, line features, and center surround features. In the most basic, edge features, the feature value of (a), (b) is represented by the difference between two sums- the sum of all pixel values in the bright part and the sum of all the pixel values in the dark areas. (c) can be obtained by the difference between the sum of all pixel values in the outside bright part and the sum of all pixel values in the central dark part, and (d) can be obtained by the difference between the sum of all pixel values in the bright part of the diagonal direction and the sum of all pixel values in the dark part[4], [5].

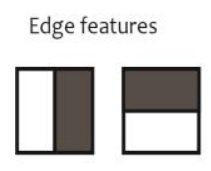

(a)

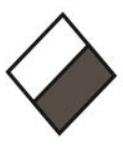

(c)

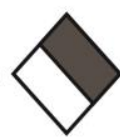

(d)

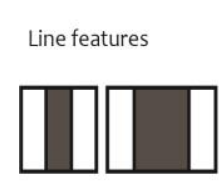

(a)

(b)

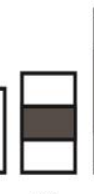

(c)

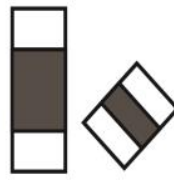

(e)

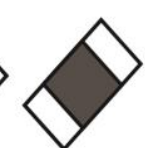

(f)

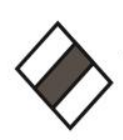

(g)

(h)

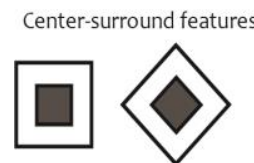

(a)

(b)

Figure 2. Feature Prototypes of Simple Haar-like and Center-surround Features Black Areas have Negative and White Areas Positive Weights 


$$
i i(x, y)=\sum_{x^{\prime} \leq x, y^{\prime} \leq y} i\left(x^{\prime}, y^{\prime}\right)_{(1)}
$$

In equation (1) $\mathrm{ii}(\mathrm{x}, \mathrm{y})$ is integral image, $\mathrm{ii}\left(\mathrm{x}^{\prime}, \mathrm{y}^{\prime}\right)$ is the brightness value of the initial image. Point $4(\mathrm{x}, \mathrm{y})$ 's value can be obtained by all cumulative pixel values to the horizontal $x$ coordinate, and to the vertical y coordinate. In figure 3 , the sum of the pixels within rectangle $\mathrm{D}$ can be computed with four array references. The value of the integral image at location 1 is the sum of the pixels in rectangle $\mathrm{A}$. The value at location 2 is $\mathrm{A}+$ $\mathrm{B}$, at location 3 is $\mathrm{A}+\mathrm{C}$, and at location 4 is $\mathrm{A}+\mathrm{B}+\mathrm{C}+\mathrm{D}$. The sum within $\mathrm{D}$ can be computed as $4+1-(2+3)[6],[7]$.

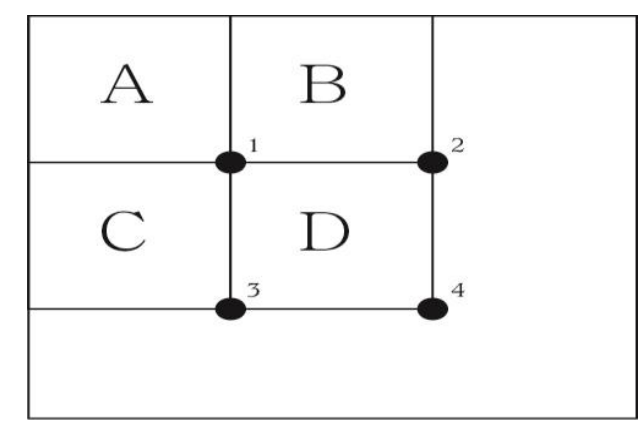

Figure 3. Rectangular Regions of an Integral Image

\section{Dual monitoring Tracking System for Children Protection}

\subsection{Abnormal Face Detection}

The algorithm called Adaboost was introduced by Freund and Schapire. Linearly combining multiple weak classifiers, the final strong classifier with the high detection performance is created. Adaboost has been applied to many classifications and recognition problems including face search, objects search, facial recognition, voice recognition, and feature selection[4].

Adaboost classifier can be learned using the set of training images which consist of face and abnormal facial pattern, image set, and feature set. Classifiers are made up to detect the abnormal face, and to remove normal face. Input vector $\mathrm{X}$ is features' set; $\mathrm{X}$ i is a wavelet feature mask. Output $Y$ has a value of +1 and -1 , and they represent the face and abnormal face respectively. Figure 4 shows the AdaBoost algorithm that was used in this paper.

$$
\begin{aligned}
& \text { 1. Given training data }\left(\mathrm{x}_{1}, \mathrm{y}_{l}\right), \ldots,\left(\mathrm{x}_{\mathrm{m}}, \mathrm{y}_{\mathrm{m}}\right) \text {, } \\
& \text { where } \mathrm{x}_{\mathrm{i}} \in \mathrm{X}, \mathrm{y}_{\mathrm{i}} \in=\{-1,+1\} \\
& \text { Initialize data weights } D_{1}(\mathrm{i})=1 / \mathrm{m} \\
& \text { 2. For } \mathrm{t}=1, \ldots, \mathrm{T} \\
& \quad \text { Train weak learner using distribution } \mathrm{D}_{t} \\
& \text { - Get weak hypothesis } \mathrm{h}_{\mathrm{t}}: \mathrm{X} \rightarrow\{-1,+1\} \text { with error. } \\
& \text { - Choose } \alpha_{t}=\frac{1}{2} \ln \left(\frac{1-\varepsilon_{t}}{\varepsilon_{t}}\right) \\
& \text { - update: }
\end{aligned}
$$




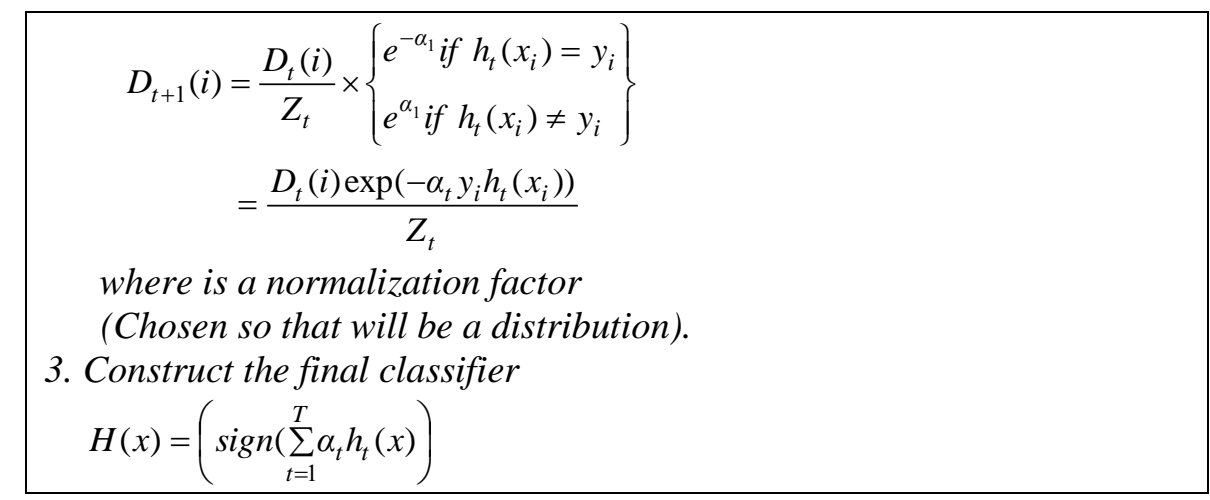

Figure 4. AdaBoost Algorithm

The weak classifier ht classifies training patterns using a wavelet mask Xi. At this time the incorrectly classified training pattern increases the weights Dt(i), while the correctly classified training patterns reduces Dt(i). When, repeating this process, the abnormal facial pattern recognition is accomplished, and the tracking and monitoring system operates through notification services and smartphones. Figure 5 shows the stages of training patterns for detecting abnormal face using AdaBoost algorithm.

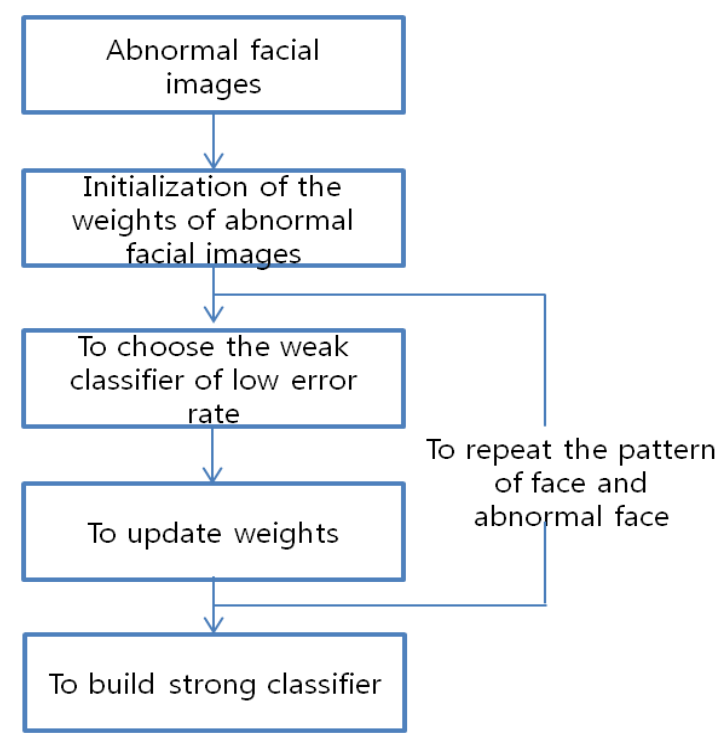

Figure 5. Training Patterns for Detecting Abnormal Face

\subsection{Tracking System}

In this paper, when tracking faces using wide-area surveillance cameras and the PTZ(Pan Tilt Zoom) camera with high magnification, the tracking was accomplished by using only the minimum amount of information including the movement orientation of the abnormal face between the frames, location information, and so on. Face tracking uses the method of moving the center of the detected area to the center of the entire image as the basic concept. 


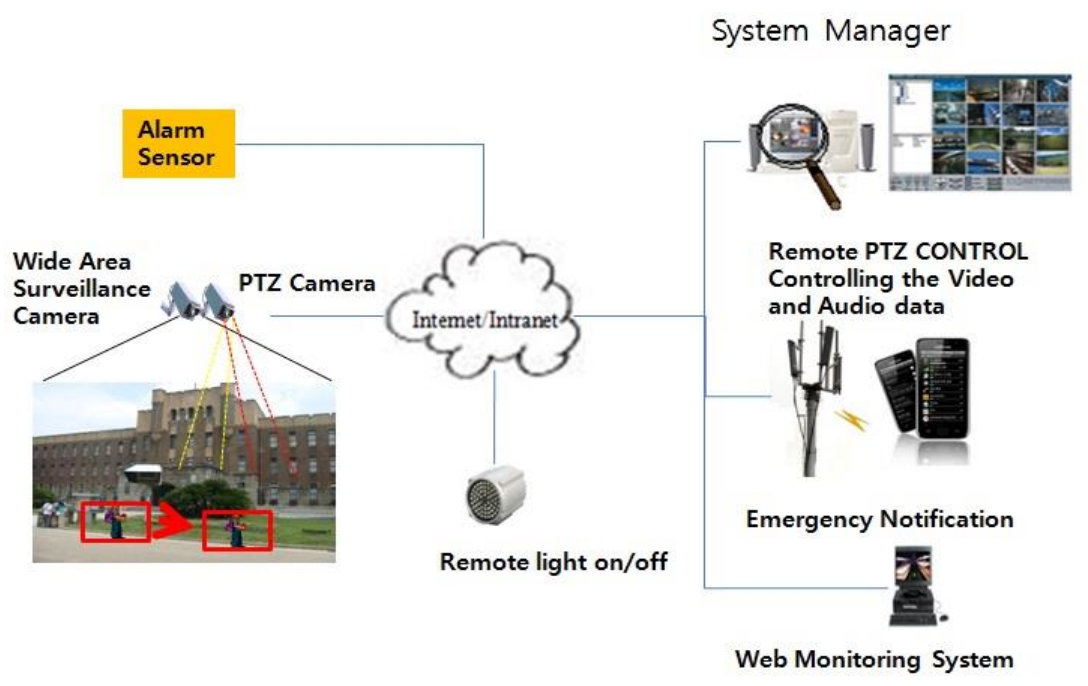

Figure 6. The Configuration of the Tracking System with Dual Monitoring

Figure 6 represents the configuration of the tracking system using wide-area surveillance cameras and the PTZ camera with high magnification. Figure 7 selected the screen's center point as (x, y) for the face tracking, and the center point of abnormal face screen selected the half of $(\mathrm{w}, \mathrm{h})$ as $\left(\mathrm{x}^{\prime}, \mathrm{y}^{\prime}\right)$, and move the monitor and track abnormal face until (x,y) will be the same. In an abnormal face's moving images, it continues to track until the face comes in the center of the camera, and the calculation of the center of fixed abnormal facial image is shown in equation (2).

$$
\left(x^{\prime}, y^{\prime}\right)=\left(\left(\frac{x+w}{2}\right) \times \text { sclae },\left(\frac{y+h}{2}\right) \times \text { sclae }\right)
$$

When it detects an abnormal face, it gives the notification signal that the abnormal situation has occurred, and carries out continuous monitoring service. To prepare for the case of tracking failure due to the failure of abnormal face detection 10-frame-face position and size information is stored.
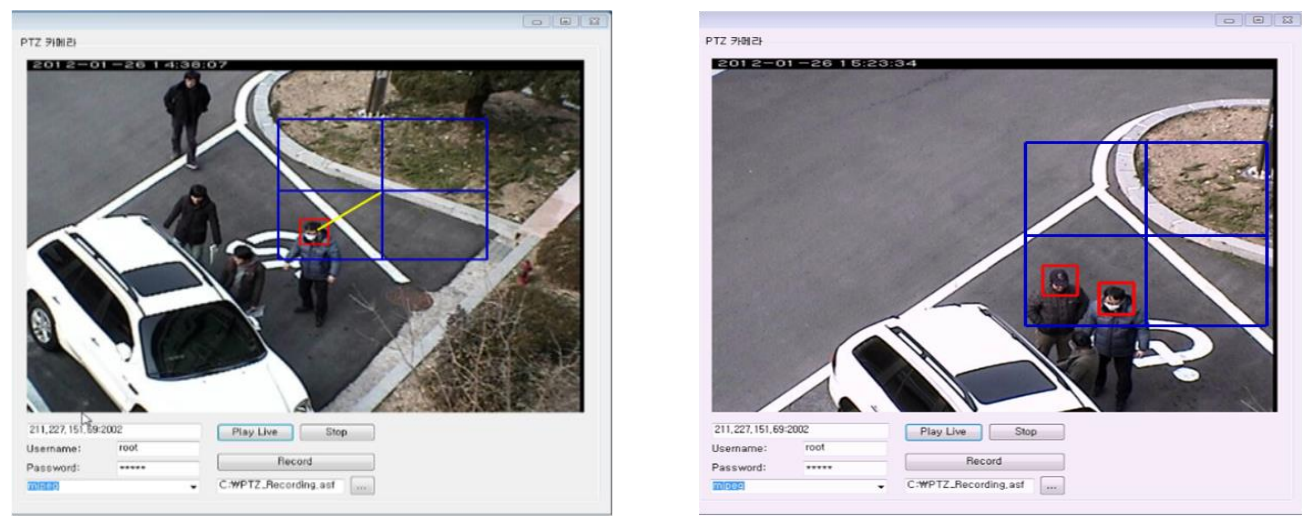

Figure 7. Faces Tracking in Wide-area Video Surveillance Camera

The system used in this experiment has used OpenCV 2.3 library in Intel i5 1.83Ghz, 4GB, Windows 7 environment by taking advantage of Visual Studio 2010. To improve the efficiency of calculations between the data of abnormal face and features they were normalized into the size of $20 * 20.6500$ abnormal data and 2700 normal face data were used. In the process of adjusting the weights in order to remove the normal facial pattern 
according to the experimental results, the face pattern that wasn't strong was removed. Table 1 represents recognition rate based on the abnormal face recognition technique. Viola Jones showed 93.5\%, Sheiderman-Kanade 93\%, Rowley 92.5\%, the proposed algorithm showed the recognition of $95.5 \%$. Table 2 represents the average time of recognition, tracking, and image storage for the processing of the abnormal face.

\section{Table 1. The Recognition Rate based on the Abnormal Face Recognition} Technique

\begin{tabular}{|l|l|}
\hline The abnormal face recognition technique & The recognition rate \\
\hline Viola-Jones & $93.5 \%$ \\
\hline Sheiderman-Kanade & $93 \%$ \\
\hline Rowley & $92.5 \%$ \\
\hline The proposed algorithm & $95.5 \%$ \\
\hline
\end{tabular}

Table 2. The Results of Tracking System of Abnormal Face

\begin{tabular}{|c|c|c|c|c|}
\hline & $\begin{array}{c}\text { Image } \\
\text { Grab }\end{array}$ & $\begin{array}{c}\text { Abnormal } \\
\text { face recognition }\end{array}$ & $\begin{array}{c}\text { Abnormal } \\
\text { face tracking }\end{array}$ & $\begin{array}{c}\text { Image } \\
\text { storage }\end{array}$ \\
\hline Processing time & $40 \mathrm{~ms}$ & $52 \mathrm{~ms}$ & $59 \mathrm{~ms}$ & $71 \mathrm{~ms}$ \\
\hline $\begin{array}{c}\text { The number of } \\
\text { frames per second }\end{array}$ & $\begin{array}{c}25 \\
\text { frame }\end{array}$ & 19 frame & 17 frame & 14 frame \\
\hline
\end{tabular}

\section{Conclusion and Future Works}

Child protection means all types of services and programs that society or adults can do for children in order to promote their healthy growth and development process. As Ministry of Education, Science and Technology and Ministry Administration and Security started operating Public CCTV around the Schools through the integrated CCTV control center of local governments, there have appeared a lot of problems. The existing security system built on saving the image has its advantages in terms of easy use and low cost maintenance, but there are the defects that the video cameras should be installed in the each main point, the images should be recorded on the VCR or DVR, and then reviewed after the event has occurred. For crime prevention managers should directly the watch the monitor for 24 hours a day. Because of this inconvenience it cannot provide the effective response to the criminal case after school or on weekends or holidays, and culprits abuse this time range. As a result, it was difficult to provide a preliminary prevention of crime.

In this paper, for the protection of child, the system tracking and monitoring abnormal face in wide-area surveillance using wide-area surveillance cameras and PTZ cameras with the high magnification was proposed. By the use of the method of moving the center of the face to the center of the whole image, abnormal face was tracked using the AdaBoost algorithm. As the result of the experiments, 95.5\% of the abnormal facial recognition rate and the average speed of 17 frames of abnormal were obtained. The problems of artificial monitoring by the people, and costly installation were complemented. Automatic detection and recognition function for abnormal situation was determined. Low installation costs can help configure a variety of remote monitoring and control systems, and it is considered that this system would be a help in protecting children from the intelligent criminal and crime prevention. 
Future works will be the development of natural disaster analysis systems such as the fire of wide area using techniques related to image analysis and shape analysis, and utilizing it in research area for endangered plants and animals.

\title{
Acknowledgement
}

This research was financially supported by the Ministry of Education (MOE) and National Research Foundation of Korea(NRF) through the Human Resource Training Project for Regional Innovation (No. 2013H1B8A2032217).

\section{References}

[1] E. Y. Kang, "How to Institutionalize CPTED In Korea (III) : Safer School and Safer Community", Korean Institute of Criminology, (2010).

[2] S. M. Cha and B. H. Lee, "Child Protection System using RTLS", Proceedings of Korea Information Processing Society, (2011).

[3] S. S. Kwak, J. Y. Jung, E. M. Shin and J. G. Choi, "Design Guidelines for childcare Robots with an Ethnographic Study of Dual Income and Single Income Families", The Bulletin of JSSD, vol. 55, (2008), pp. 65-74.

[4] R. E. Schapire, "The Boosting Approach to Machine Learning an Overview", MSRI Workshop on Nonlinear Estimation and Classification, (2002), pp. 1-23.

[5] W. J. Lee, J. C. Kim and B. H. Lee, "Real-time Face Detection and Tracking using the AdaBoost Algorithm", Journal of Korea Multimedia Society, vol. 9, (2006), pp. 1266-1275.

[6] P. Viola and M. J. Jones, "Robust Real-Time Face Detection", International Journal of Computer Vision, vol. 57, (2004), pp. 137-154.

[7] P. Viola and M. Jones, "Rapid Object Detection using a boosted cascade of simple features", Conference on computer Vision and Pattern Recognition, (2001).

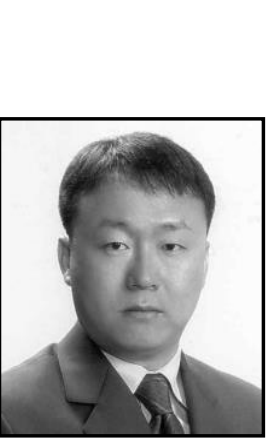

\begin{abstract}
Authors
Jong Chan Kim, he received his B.S. degree from Sunchon National University in 2000, his M.S degree from department of computer science, Sunchon National University in 2002, his Ph.D. degree from department of computer science, Sunchon National University in 2007. And a senior researcher professor of the Automation and System Research Institute at Seoul National University in 2013. His current research interests are image processing, computer graphics, digital clothing, AR, games and HCI.
\end{abstract}

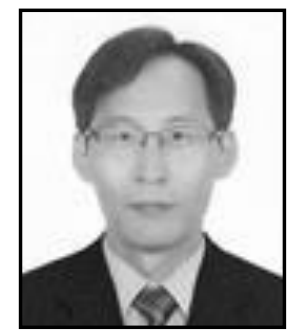

Oh Hoon Cho, he received the B.S. degree from Korea National Open University, Korea, in 2006, His M.S. degree from department of computer science, Sunchon National University, Korea, in 2013. His currently a Ph.D. student in computer science at the Sunchon National University, Korea, His current research interests include augmented reality, image processing, computer graphics. 


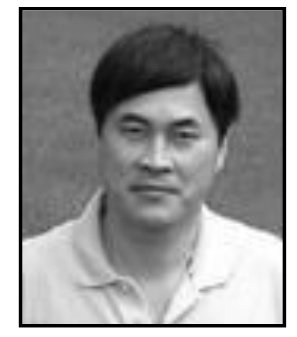

Eung Kon Kim, he received the B.S. degree from Chosun University, Gwangju,, Korea, in 1980, his M.S degree from department of electronics, Hanyang University, Seoul, Korea, in 1987, his Ph.D. degree from Chosun University, Gwangju, Korea, in 1992. His current research interests are computer vision, virtual/augmented reality, image processing, and computer graphics. Currently he is a professor in department of computer engineering, Sunchon National University, Korea.

*Corresponding author: Eung Kon Kim, Ph.D.

Department of Computer Science, Sunchon National University 540-742, 315 Maegok,

Suncheon, Jeonnam, Korea

E-mail:kek@sunchon.ac.kr 
International Journal of Control and Automation Vol. 8, No.4 (2015) 